\title{
Modelos de canales abiertos considerando el intercambio de energía con el medio ambiente y el transporte de contaminantes
}

\section{Open channel models that consider the exchange of energy with the environment and the transport of pollutants}

Presentación: 00/00/0000

\section{Doctorando:}

\section{Evangelina S. Delfratte}

CAIMI (Universidad Tecnológica Nacional - Facultad Regional Rosario)

evangelinadelfratte@yahoo.com.ar

\section{Director/es:}

\section{Alejandro S. M. Santa Cruz \\ Nicolás J. Scenna}

\begin{abstract}
Resumen
Se implementa un modelo que representa en forma simplificada una corriente superficial de agua, ya sea natural o artificial, sobre la que se simula un vertido de una sustancia contaminante de características conservativas. Se comparan los resultados obtenidos para diferentes escenarios (ancho del canal, rugosidad, concentración inicial, pendiente del lecho) con los obtenidos con un software de uso libre (HEC RAS). Por otra parte, se analiza la influencia de la temperatura ambiente sobre la temperatura del curso de agua, así como la representación de las variables estocásticas de mayor influencia en los resultados utilizando funciones de distribución.
\end{abstract}

Palabras claves: canal 1D, función de densidad de probabilidad de caudales

\begin{abstract}
The implementation of a simplified surface water stream model, whether natural or artificial, on which the discharge of a conservative characteristics substance (contaminant) is simulated is carried out. The results of different case-scenario (channel width, roughness, initial concentration, bed slope) are compared with those obtained with free software (HEC RAS). On the other hand, the influence of ambient temperature on the course temperature is modelled, as well as the stochastic variables with higher influence on the results by means of distribution functions.
\end{abstract}

Keywords: 1D channel, flow probability density function.

\section{Introducción y metodología}

Históricamente, investigadores se han interesado en modelar el transporte de contaminantes que podrían poner en peligro el ecosistema y la salud de las personas si fueran liberados a un curso de agua. En la mayoría de los casos, el interés se centra en pronosticar la concentración máxima que alcanzará en lugares particulares, el tiempo de llegada del pico y la ocurrencia y duración de niveles peligrosos de contaminantes.

Con el fin de obtener una base de cálculo para la obtención de índices de riesgo relativos a los derrames de sustancias y su distancia de afectación e impacto, se implementó en GAMS [1] un modelo matemático que representa la circulación de un fluido (agua) por un canal abierto y el perfil de contaminante en distancia y tiempo, supuesto un vertido de sustancia en concentración y volumen conocido.

El sistema de ecuaciones para representar un canal con secciones transversales variables y un flujo constante gradualmente variado viene dado por las ecuaciones (1-2), donde $h$ representa el nivel de agua por encima del dato asumido, $Q$ es la velocidad de flujo, $q$ es la entrada lateral, $x$ es la distancia longitudinal, $Z$ es el lecho de elevación, $\beta$ es 
el factor de corrección de energía y $A$ es el área de la sección transversal, $E$ representa la energía total de la corriente que fluye [2].

$$
\begin{aligned}
& \frac{d Q}{d x}=q \\
& \frac{d E}{d x}=\frac{\partial}{\partial x}\left(h+\frac{\beta \cdot Q^{2}}{2 g \cdot A^{2}}\right)=-S-\frac{\beta \cdot Q}{g \cdot A^{2}} q
\end{aligned}
$$

La ecuación (3) describe el transporte de advección-difusión 1D de un contaminante conservativo (trazador) disuelto en agua para un canal abierto no prismático, donde C, x y t se refieren a la concentración, distancia y tiempo, respectivamente.

$$
C \frac{\partial A}{\partial t}+A \frac{\partial C}{\partial t}+A \frac{\partial Q}{\partial x}+Q \frac{\partial C}{\partial x}-\frac{\partial}{\partial x}\left(A D \frac{\partial C}{\partial x}\right)+A \delta=0
$$

El balance de calor dependiente del tiempo se da en la Ecuación (4); en tanto que la temperatura de la corriente de agua (T) depende del calor neto intercambiado con el ambiente y el sedimento, tal como se expresa en la Ecuación (5).

$$
\begin{aligned}
& \rho C_{p}\left(\frac{\partial T}{\partial t}+v_{x} \frac{\partial T}{\partial x}\right)=k\left(\frac{\partial^{2} T}{\partial x^{2}}\right)+Q_{N e t} \\
& Q_{\text {Net }}=J_{\text {snt }}+J_{\text {longat }}-J_{\text {back }}-J_{\text {conv }}-J_{\text {evap }}+J_{\text {sed }}
\end{aligned}
$$

$$
\begin{aligned}
& \rho C_{p} \frac{\partial T}{\partial t} \\
& \rho C_{p} v_{x} \frac{\partial T}{\partial x} \\
& k\left(\frac{\partial^{2} T}{\partial x^{2}}\right) \\
& \mathrm{Q}_{\mathrm{Net}}
\end{aligned}
$$

Velocidad de incremento de la temperatura del curso de agua por unidad de volumen

Velocidad de incremento de la temperatura del curso de agua debido a la convección por unidad de volumen

Velocidad de incremento de la energía interna debido a la conducción por unidad de volumen Velocidad de adición de energía debido a fuentes externas por unidad de volumen

Los flujos de calor considerados son: radiación solar de onda corta (J $\left.\mathrm{J}_{\mathrm{snt}}\right)$, radiación atmosférica de onda larga (Jlongat), radiación de onda larga emitida por el agua (J back), convección (J conv), evaporación y condensación (Jevap), conducción con los sedimentos (J Jed).

Se llevó a cabo la representación de una serie de canales rectos (en su longitud) variando la sección transversal (trapezoidal constante y creciente, rectangular constante y creciente), la pendiente e inclinación del talud. Los resultados obtenidos por el modelo implementado en GAMS han sido validados por comparación con los obtenidos al emplear el software HEC RAS (U.S. Army Corps of Engineers' River Analysis System) [3].

Además de lo anterior, se ha estudiado la dinámica de transferencia de calor del curso de agua y su entorno. Por medio de un modelo dinámico que permite predecir el perfil de temperatura de la corriente de agua en estudio, así como el intercambio con el lecho, al considerar cada flujo importante de calor que impacta en el sistema (incluyendo términos de radiación y convección, evaporación y condensación). Los resultados se comparan con los obtenidos mediante del módulo rTemp incluido en QUAL2Kw [4], que es un algoritmo reconocido programado en Excel para analizar la calidad del agua en ríos y arroyos.

Finalmente, considerando un caso de referencia detallado en Tabla 1, se analizó la variación en la altura del agua y el perfil de concentración de contaminantes por medio de la simulación paramétrica de 40 casos de estudio.

Tabla 1. Estudio de influencia de parámetros sobre resultados. Caso de referencia

\begin{tabular}{lcccc}
\hline & pendiente del lecho [--] & Ancho del canal $[\mathrm{m}]$ & Rugosidad $[--]$ & Cmax $\left[\mathrm{g} / \mathrm{m}^{3}\right]$ \\
\hline Parámetros & 0.0036 & 12.5 & 0.08 & 61.7 \\
\hline
\end{tabular}

Se ejecutaron múltiples simulaciones, en las que cada valor de parámetro cambia individualmente mientras se mantienen constantes los demás, considerándose el impacto del parámetro CT sobre el valor de concentración máxima. Se variaron el valor de la pendiente (de 0.00290 a 0.00390 ), el ancho del canal en un rango de $6.20 \mathrm{~m}$ a $18.70 \mathrm{~m}$, la rugosidad desde 0.0250 a 0.1375 (con incrementos de 0.0125 ) y CT en el rango de 30 a 50. 
En relación a la obtención de funciones de distribución de caudales, se utilizaron datos publicados por la Subsecretaría de Recursos Hídricos de la Nación [5]: series históricas de aforos, caudales y alturas de algunos cursos fluviales de la Argentina para identificar tipos de función de distribución que representen la variación de caudal en cauces naturales. Analizando, en los casos en los que se dispone de numerosas mediciones diarias, la amplitud registrada para cada día. En todos los casos, se realizó la estimación de los principales parámetros estadísticos y se propusieron funciones de distribución de tipo Normal, Log-Normal, Gumbel y Gamma. Como prueba de ajuste se realizó el test Kolgomorov-Smirnov para tres niveles de significación [6].

\section{Resultados}

Los resultados obtenidos a partir del modelo propuesto se compararon con los correspondientes al software HEC RAS. Se variaron paramétricamente las variables asociadas al caudal del cauce y vertido de acuerdo a su naturaleza estocástica. Si bien se analizaron una gran cantidad de variaciones en los parámetros (pendiente del lecho, ancho del canal, rugosidad, CT, etc.) a continuación se representan a) la sección transversal inicial ( $\mathrm{L}=0 \mathrm{~m})$ y final ( $\mathrm{L}=2000 \mathrm{~m})$, b) la sección transversal ocupada y c) la variación del Número de Froude a lo largo del canal (Figura 1, Figura 2 y Figura 3, respectivamente) para uno de los casos considerados. En las últimas dos figuras se observa claramente que los resultados son similares a los obtenidos con el software HEC RAS.

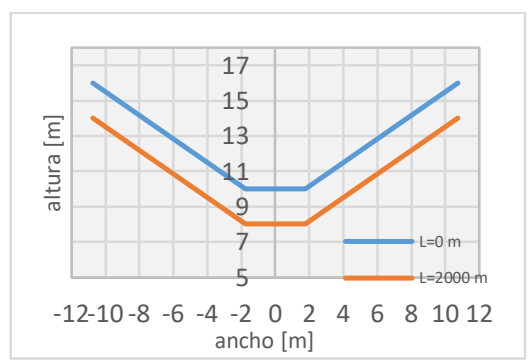

Figura 1. Sección transversal inicial y final

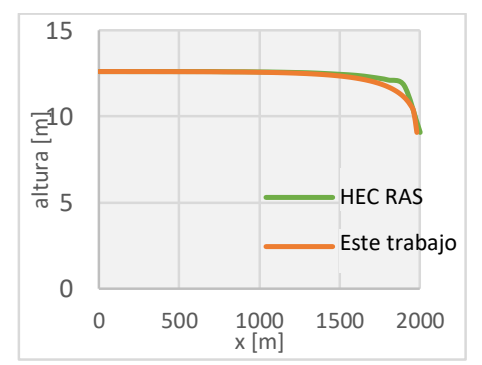

Figura 2. Sección transversal ocupada

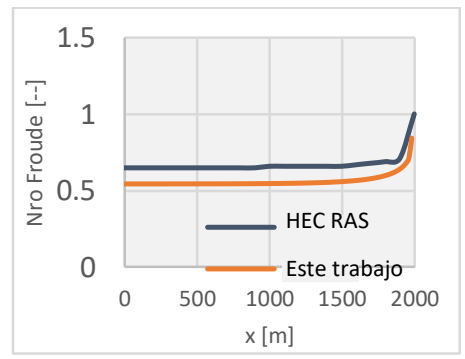

Figura 3. Variación del número de Froude

Para la simulación de la dinámica del curso de agua, se asume un canal con flujo predominante en una dirección única de circulación y de dimensiones tales que permite el mezclado completo de su sección transversal.

Los datos de entrada contemplan los parámetros y propiedades del sistema (densidad, calor específico, conductividad, calor latente, entre otros), temperatura del aire, temperatura de rocío, velocidad del viento, fracción cubierta por nubes y radiación solar recibida en la capa externa de la atmósfera. Se dispone de esta información cada 15 minutos, siendo considerados constantes durante ese lapso de tiempo. Se obtienen los flujos de la radiación solar de onda corta, radiación atmosférica de onda larga, radiación solar de onda larga proveniente del cuerpo de agua, evaporación y condensación, convección e intercambio con sedimentos así como los perfiles de temperatura del curso de agua y de los sedimentos. En particular, en la Figura 4 se representa, para una ubicación definida, el flujo de calor asociado a procesos convectivos, durante 6 días (cada día se representa por un período de 1.440 minutos en hora local) mientras que la Figura 5 muestra el perfil de temperaturas del curso de agua. El perfil de temperaturas del sedimento (no mostrado) es similar al del curso de agua. [7]

Los resultados (representados en azul) se comparan con los obtenidos mediante el módulo rTemp. Es necesario resaltar aquí que el coeficiente de correlación $\left(R^{2}\right)$ para la mayoría de las variables analizadas es mayor a 0.95 , poniendo de manifiesto el buen nivel de aproximación del modelo propuesto en este trabajo.

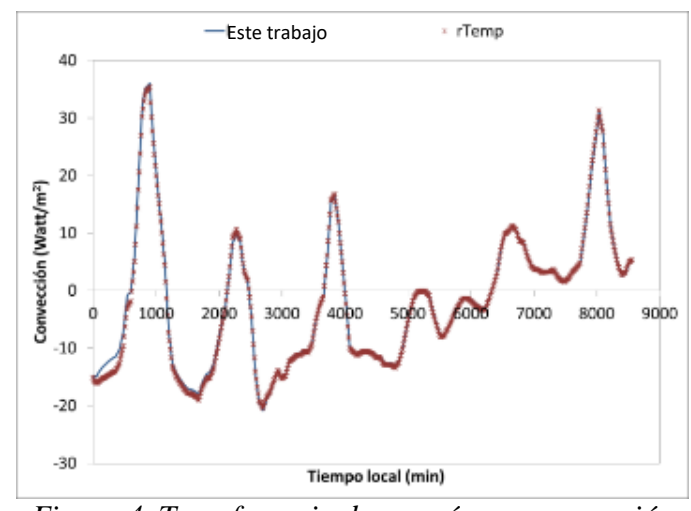

Figura 4. Transferencia de energía por convección

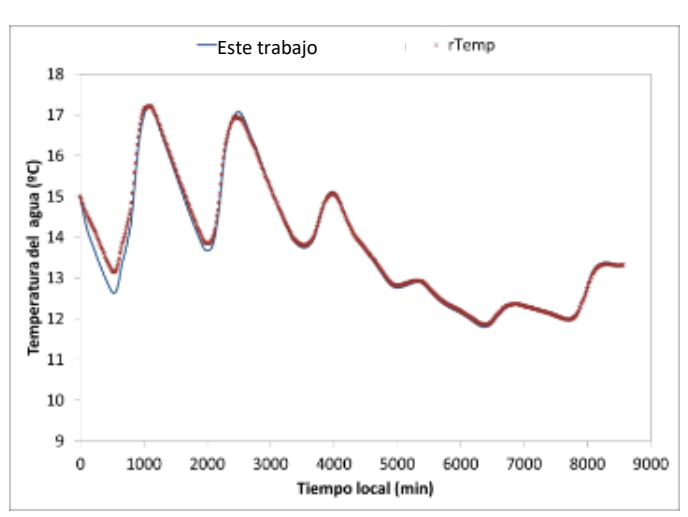

Figura 5. Temperatura del curso de agua 
Por otro lado, las Figuras Figura 6 a Figura 8 muestran la influencia de la variación del ancho del canal en el nivel de profundidad y el flujo de agua, en el área transversal y la pendiente de fricción y en la concentración [8]. El modelo propuesto para la estimación de la altura del agua exhibe un rendimiento aceptable en comparación con el software disponible, permitiendo determinar los parámetros principales que afectan el comportamiento del sistema, al tiempo que ofrece estimaciones precisas de la evolución del perfil del trazador para el curso de agua considerado.

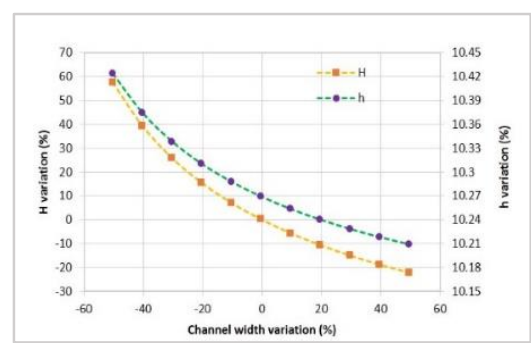

Figura 6. Variación del nivel de profundidad de agua $(H)$ y flujo $(h)$

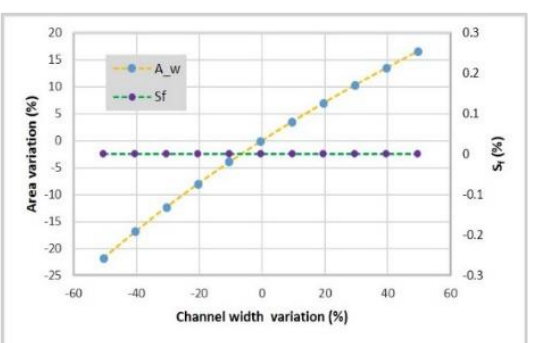

Figura 7. Variación del área transversal y pendiente de fricción

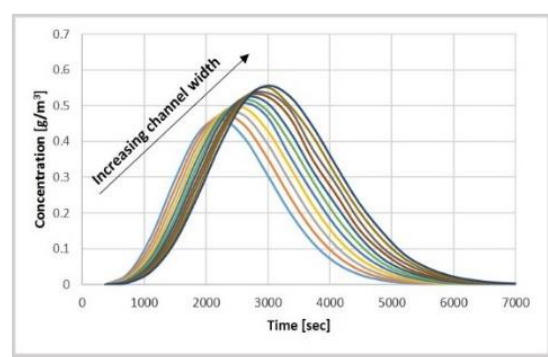

Figura 8. Variación de la concentración

Finalmente, en las Fig. 9 a 12 se representa la variación de la concentración máxima de contaminante frente a variaciones en la pendiente del lecho, el ancho del canal, la rugosidad y la concentración inicial aguas arriba [8]. Con respecto al perfil de concentración del marcador, se observa que las variaciones en el ancho del canal (Fig. 10) y en la concentración inicial (Fig. 11) del marcador producen las mayores diferencias respecto del caso de referencia. Por otro lado, las variaciones la pendiente del lecho (Fig. 9) y rugosidad (Fig. 12) no afectan significativamente los resultados observados. Sin embargo, un aumento en la rugosidad del canal produce una curva de perfil de concentración cada vez mayor, así como un retraso en la concentración máxima del marcador (Fig. 8).

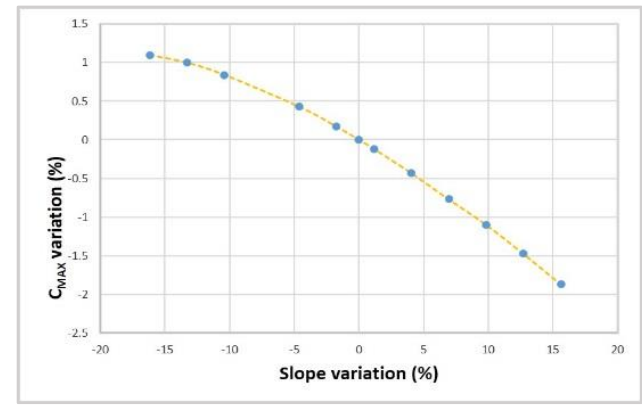

Figura 9. Concentración máxima frente a la variación de la pendiente del lecho

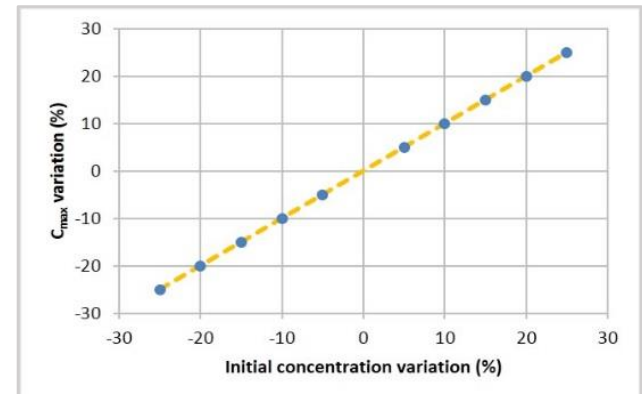

Figura 11. Concentración máxima frente a la variación de la concentración inicial aguas arriba

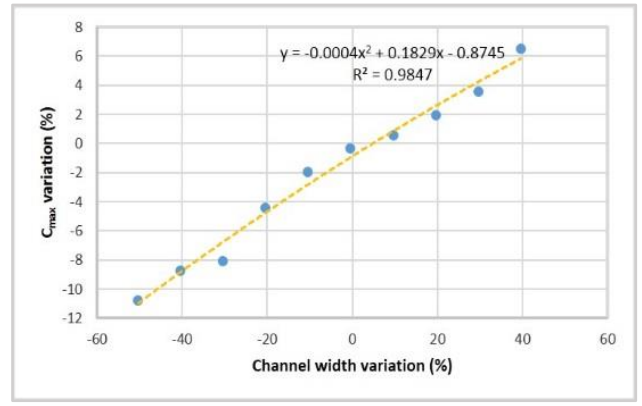

Figura 10. Concentración máxima frente a la variación del ancho del canal

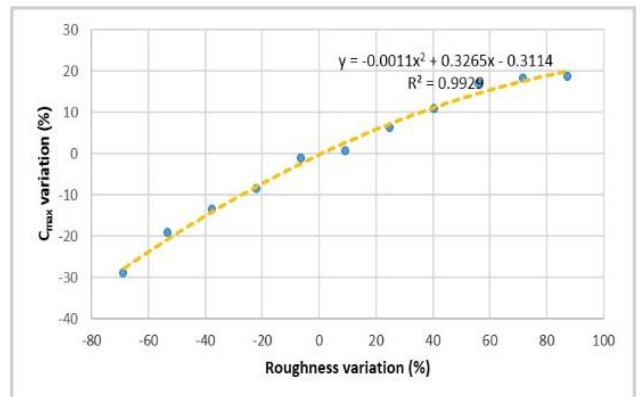

Figura 12. Concentración máxima frente a la variación de la rugosidad

En relación a las funciones de distribución de caudales, hasta el momento se obtuvieron resultados de 8 estaciones de distintos cauces. De estas, la mitad podría ser representada al menos con una de las funciones de distribución propuestas, con un $\alpha=0.05$ para el test de Kolgomorov-Smirnov. Tres de las estaciones responden a un ajuste a distribución Log-Normal, dos a Gamma y una a Normal.

\section{Referencias}

[1] GAMS. A User's Guide. Tutorial by Richard E. Rosenthal. GAMS Development Corporation, Washington, DC, USA. January 2012. 
[2] Szymkiewicz, R. (2010). Numerical Modeling in Open Channel Hydraulics, Faculty of Civil and Environmental Engineering, Gdansk University of Technology, Poland. Springer.

[3] HEC-RAS River Analysis System. Hydraulic Reference Manual. Version 4.1. January 2010.

[4] PELLETIER, G.; CHAPRA, S. (2004). QUAL2Kw theory and documentation - A modeling framework for simulating river and stream water quality. Washington. Washington State Department of Ecology,

[5] Base de Datos Hidrológica Integrada de la Secretaría de Infraestructura y Política Hídrica de la Nación. http://bdhi.hidricosargentina.gob.ar/

[6] Canavo, G. (1998). PROBABILIDAD Y ESTADISTICA, Aplicaciones y métodos. McGraw Hill. ISBN 968-451-856-0

[7] Delfratte, E. (2014). Modelo Térmico 1D de un curso de agua, considerando factores ambientales-atmosféricos. Jornadas de Jóvenes Investigadores Tecnológicos 2014 - 1 a ed. - Rosario: Universidad Tecnológica Nacional. Facultad Regional Rosario. Secretaría de Ciencia y Tecnología. 2015. E-Book. ISBN 978-950-42-0162-5 1. Actas de Congreso.

[8] Delfratte, E. Scenna, N. Santa Cruz, A. (2016). Sensitivity analysis of water stage and pollutant concentration profiles for an open-channel course. EngOpt 2016 - 5th International Conference of Engineering Optimization. - ISBN 978-85-7650-522-8 